\title{
Fasting Therapy for Psychosomatic Diseases with Special Reference to Its Indication and Therapeutic Mechanism
}

\author{
Jinichi Suzuki, Yuichi Yamauchi, Masatoshi HoRIkawa \\ and Shotchi Yamagata \\ Department of Psychosomatic Medicine, Nagamachi Branch Hospital, \\ Tohoku University School of Medicine, and The Third Department \\ of Internal Medicine, Tohoku University School of Medicine, Sendai
}

Suzuki, J., Yamauchi, Y., Horikawa, M. and Yamagata, S. Fasting Therapy for Psychosomatic Diseases with Special Reference to Its Indication and Therapeutic Mechanism. Tohoku J. exp. Med., 1976, 118 (Suppl.), 245-259It has been well known that the fasting therapy which was invented in Medical School of Tohoku University reveals an excellent effect upon various kinds of psychosomatic diseases, but its therapeutic mechanism and suitable indication are not yet explained completely. In order to corroborate these problems, this study was undertaken on 262 cases of psychosomatic diseases in the field of internal medicine. It is a complete fasting for 10 days with nothing by mouth except for drinking water, and $500 \mathrm{ml}$ of parenteral fluid containing vitamins are administered intravenously every day. Absolute bed rest and self meditation are required in a closed individual room, and patients are not allowed to meet anyone but physician and nurse in charge. The return to normal ordinary diet follows the order of fluid diet, soft diet and semiordinary diet during 5 days. In the period of the therapy, various clinical and laboratory examinations were carried out. Significance of these examinations consists in prediction of possible danger during the fasting period and elucidation of its therapeutic mechanism. Consequently, an outstanding efficacy rate of $87 \%$ with excellent prognosis was attained, and the following diseases were determined as suitable indication of this therapy; irritable colon, dysorexia nervosa, borderline hypertension, neurocirculatory asthenia, bronchial asthma, mild diabetes mellitus, obesity, lumbago without organic findings, conversion hysteria, various neurosis with somatic symptoms and masked depression. Possible mechanism of action of the therapy is that fasting acts as an extreme stress on the function of the autonomic nervous and endocrine systems, then it regulates the function of whole body including the brain, also it acts as one of the behaviour therapy for abnormal conditioning. fasting therapy; psychosomatic disease; EEG; growth hormone; behavior therapy

Total fasting has been practised by layman for thousands of years mainly for religious purposes in both the Western and Oriental countries. Recently, however, the fasting has become increasingly a common method for treating

Received for publication, November 20, 1975 .

Address for reprints: Jinichi Suzuki, M.D., Department of Psychosomatic Medicine, Nagamachi Branch Hospital, Tohoku University School of Medicine, 13-1, 5 Nagamachi, Sendai 982, Japan. 
obesity in Western countries (Bloom 1959; Duncan et al. 1963; Drenick et al. 1964; Thomson et al. 1966). In Japan, an attempt was made to study the reaction of the living body to fasting from the morphological, physiological and biochemical viewpoints by Takahira (1930) who pointed out the clinical effectiveness of this therapy in treating various chronic diseases. Kushima et al. (1961) and Hasegawa (1961) noted the disappearance of somatic and mental symptoms in response to fasting. They applied it to gynecological patients with neurosis, autonomic nerve dystonia, etc. The results of the fasting therapy showed a surprisingly high efficacy rate of $90 \%$. Through the series of reports about fasting therapy, such biochemical changes as ACTH, adrenocortical hormones, sex hormones and serum serotonin levels were observed during and after the fasting process (Ogawa 1958; Kushima et al. 1959; Yamauchi 1959). These changes of the peripheral substances will necessarily reflect the functions of central nervous systems (CNS), especially of the portions around limbic and thalamo-hypothalamic systems. It is of value, therefore, to pursue the pathophysiology of fasting. The authors have tried and extended this therapy to many psychosomatic patients in the field of internal medicine in the past 7 years. The results of our new method of fasting therapy were satisfactory, as compared with those in the previous reports.

The method used here is somewhat different from that of Kushima-Hasegawa, because small amounts of pentose solution were parenterally administered in order to prevent dehydration and liver dysfunction during fasting. Augmenting the original method in this way, however, had no adverse effect on the therapeutic value of fasting. In our experience, fasting therapy has dramatic effects on the psychic aspects of the patients, possibly by reorganizing the normal psychosomatic interaction by loading strong physical stress. However, little is known about the actual mechanism of this therapeutic effect. Accordingly, some kinds of clinical and laboratory examinations were carried out in order to clarify the therapeutic mechanism and to determine the clinical indication.

\section{SưBJECTS AND Methods}

The fasting therapy was performed on 262 patients with psychsomatic and mental diseases of various types. About $2 / 3$ of the patients suffered from psychosomatic diseases. These diseases were:

Digestive disorders. Irritiable colon, polysurgery cases, functional disturbance of upper GI, aerophagia, postcholecystectomy syndrome, gastrointestinal neurosis.

Endocrine and metabolic disorders. Diabetes mellitus of mild degrees, anorexia nervosa, obesity, hyperthyroidism.

Muscle and skeletal disorders. Tension headache, migraine, so-called lumbago or myalgia, tic, spasmodic torticollis, SMON, angioneurotic edema.

Circulatory disorders. Neurocirculatory asthenia (NCA), cardiac neurosis, mild coronary insufficiency, labile hypertension, hypotension, sinus arrhythmia, WPW syndrome, extrasystoles.

Respiratory disorders. Bronchial asthma, hyperventilation syndrome, hiceup, coughing tic, abnormal voice attack.

Vegetosis. Climacteric disturbances, psychogenic autonomic nerve dystonia.

Urinary disorders. Neurogenic bladder, enuresis. 
In the cases of mental diseases, depression and neuroses of various types, such as anxiety neurosis, conversion hysteria, hypochondriasis and obsessive-compulsive neurosis were included. As the treatment was successfully carried out in patients between 13 and 63 years of age, the age of patients generally presents no significant problem. However, aged patients with various geriatric bodily conditions were carefully handled because of poor reserve capacity of vital energy.

The effect of fasting therapy is deeply influenced by the skillfulness of initial motivation required for the therapist. The patient was at first made to read a description of impressions on fasting by other patients, or to listen to the story of other patients who successfully completed fasting. Complete routine physical examinations and laboratory tests were usually carried out during 1-2 weeks after hospitalization. Surveying the psychosocial environments of the patient, motivation for the fasting could be established following a sufficient rapport with the physician.

The method of fasting therapy consists of complete fasting for 10 days with subsequent resumption of regular meals for 5 days. In the course of the therapy, drinking of water was freely allowed and the patients were requested to take at least 1,000-1,500 ml daily. Besides, $500-1,000 \mathrm{ml}$ of $5 \%$ pentose solution with various kinds of vitamins and small amounts of amino acids were given intravenously. Throughout the full term of fasting, the patient is not allowed to meet any one such as families and visitors, thus the patient must come to grip with his problem without outside assistance. For this reason, he is usually given an individual room, where newspapers, magazines, radio and TV were prohibited. Recently, however, a "self-reflection method" has been added during the fasting period in order to improve the psychosomatic symptoms derived from abnormal personality of the patient. The principle of this therapy consists in the reflection and insight into the disorted reaction and attitude in the past toward the families of the patients. In other words, this analysis is initiated and carried out by the patients themselves (Yoshimoto 1965). Though the patients wish very much to eat at the end of fasting, nothing but the recouped diet for recovery stage should be given for 5 days. The return to the normal diet follows the order of liquid diet, $30 \%$ rice gruel, $50 \%$ rice gruel, $70 \%$ rice gruel, $100 \%$ rice gruel and normally cooked rice. Side dishes also correspond to the liquid diet. Our menu was shown in Table 1. In the case of some patients, changes in the levels of serum growth hormone (GH), of urinary catecholamines (CA) and EEG as well as routine laboratory examinations were examined during the fasting in order to clarify the psychophysiological mechanism of the fasting therapy. Urinary CA was measured according to the modified method of Yoshinaga (1960) and Sato (1971). Serum GH was determined by means of intravenous injection of $75 \mathrm{mg}$ of L-DOPA, following the procedure described by Cavagnini et al. (1972). The assay was immunologically done by the double antibody method.

TABLE 1. Menu during recovery phase

\begin{tabular}{|c|c|c|c|}
\hline & Breakfast & Lunch & Supper \\
\hline Day lst & None & $\begin{array}{l}\text { Rice-soup } 100 \mathrm{ml} \\
\text { One pickled plum }\end{array}$ & $\begin{array}{l}\text { Rice-soup } 150 \mathrm{ml} \\
\text { One pickled plum }\end{array}$ \\
\hline Day 2nd & None & $\begin{array}{l}200 \mathrm{ml} \text { of } 70 \% \text { soft-cooked rice } \\
\text { One pickled plum } \\
\text { Ordinary milk } 180 \mathrm{ml}\end{array}$ & $\begin{array}{l}200 \mathrm{ml} \text { of } 70 \% \text { soft-cooked rice } \\
\text { One pickled plum } \\
\text { Clear bean cake soup }\end{array}$ \\
\hline Day 3rd & Milk $180 \mathrm{ml}$ & $\begin{array}{l}250 \mathrm{ml} \text { of } 50 \% \text { soft-cooked rice } \\
\text { One pickled plum } \\
\text { Clear vegetable soup }\end{array}$ & $\begin{array}{l}300 \mathrm{ml} \text { of } 50 \% \text { soft-cooked rice } \\
\text { One pickled plum } \\
\text { Bean curd soup }\end{array}$ \\
\hline Day 4th & Milk $180 \mathrm{ml}$ & $\begin{array}{l}250 \mathrm{ml} \text { of soft cooked rice } \\
\text { Bean curd soup } \\
\text { Boiled vegetables }\end{array}$ & $\begin{array}{l}300 \mathrm{ml} \text { of soft-cooked rice } \\
\text { Bean curd soup } \\
\text { Boiled fish }\end{array}$ \\
\hline Day 5th & & One half of hospital food & \\
\hline Day 6th & & Ordinary hospital food & \\
\hline
\end{tabular}


The EEG examination was carried out by means of a portable 2-channel or regular 12 channel induction method, with which checks were carried out not only for the qualitative changes of EEG, such as burst of abnormal waves, but also for the quantitative changes, such as those in its frequency and amplitude. The analysis of the EEG patterns was manually done with those EEG-charts taken in one minute of induction to left occipital region at waking state with closed eyes.

\section{Results}

Therapeutic results and prognosis

Therapeutic results at the time of discharge after the end of fasting performed on 262 cases were as follows. As shown in Table 2, this therapy has proved to be $100 \%$ effective in neurogenic bladder; it has also proved to be significantly effective in irritable colon including polysurgery cases, NCA, labile hypertension and autonomic nerve dystonia. Most of these cases were initially affected or reinforced later by an undesirable conditioning mechanism. This therapy further proved to be relatively effective in: diabetes mellitus of mild degrees associated with anxiety and depressiveness, secondary anorexia nervosa, lumbago with conversion reaction and bronchial asthma. Of the cases with mental diseases, this therapy was found to be effective in conversion hysteria, anxiety neurosis and masked depression. A summary of the results indicated that an outstanding efficacy rate of $87 \%$ was attained, including $24 \%$ rating "excellent", $63 \%$ rating "good", and 13\% rating "poor". These clinical effects in total cases are shown in Table 2, where "excellent" means entire disappearance of patients' symptoms and return to the society immediately after discharge; "good" means positive effect with some remaining symptoms which require no more medical treatments. Of all the patients, prognostic study was carried out with questionnaires to 230 patients who were discharged from our hospital more than 6 months ago. Replies were received from 201 patients $(87 \%$ ). Of these patients, in recurrence $17 \%$ was

TABLE 2. Clinical effects of fasting therapy in 262 cases

\begin{tabular}{lc}
\multicolumn{2}{c}{ in 262 cases } \\
\hline Psychosomatic diseases & $100 \%$ \\
1) Urinary disorders (5) & 96 \\
2) Digestive disorders (47) & 96 \\
3) Circulatory disorders (23) & 94 \\
4) Vegetosis (16) & 93 \\
5) Metabolic disorders (27) & 76 \\
6) Respiratory disorders (17) & 75 \\
7) Neurological disorders (24) & 86 \\
Mental diseases & 82 \\
1) Depression etc. (36) & $87 \%$ \\
2) Neurosis of various types \\
Total results: Excellent (63) \\
Good (165) \\
No effect (34)
\end{tabular}


noted from each of the groups of "excellent" and "good" efficacy, while it was also found out that, of the group with "poor" efficacy, $46 \%$ was relieved from their symptoms and could return to their social lives. Hence, the prognostic evaluation of the efficacy of the fasting therapy turned out to be $78 \%$ as is shown in Table 3 .

TABLE 3. Prognostic evaluation of fasting therapy after 6 months $\sim 6.5$ years in 201 cases

\begin{tabular}{lccc}
\hline & \multicolumn{3}{c}{ Prognosis } \\
\cline { 2 - 4 } At discharge & $\begin{array}{c}\text { Excellent } \\
(\%)\end{array}$ & $\begin{array}{c}\text { Good } \\
(\%)\end{array}$ & $\begin{array}{c}\text { Ineffective } \\
(\%)\end{array}$ \\
\hline Excellent (46) & 59 & 44 & 17 \\
Good (127) & 51 & 32 & 17 \\
Ineffective (28) & 39 & 7 & 54 \\
\hline$(\quad$ ) Number of cases & &
\end{tabular}

\section{Changes in bodily functions}

Body weight, blood pressure and heart rate. It is natural that body weight decreases gradually during fasting. When fasting terminated, a decrease of $3-6 \mathrm{~kg}$ in body weight was usually observed. The average decrease by $13.2 \%$ was of course without danger. Mcet sf the patients restored the initial body weight within about 2 weeks after resuming diet.

Systolic blood pressure fell from the average of $126 \pm 26$ to $108 \pm 12 \mathrm{mmHg}$ in the intermediate period of fasting. Thereafter, scarcely any fluctuation was noted until the 10th day of fasting. No significant changes in diastolic blood pressure was observed throughout the whole period. The systolic blood pressure once decreased and did not easily return to the previous level even in hypertensive patients.

Pulse rate significantly increased by about 14 per minute during the intermediate stage of fasting, but returned to the previous level after resuming diet. Summarized results of them were indicated in Fig. 1.

$E C G$. In psychosomatic patients with circulatory disorders, especially in the cases of NCA or borderline hypertension who exhibited no ECG abnormality in response to exercise test, ST-T abnormalities and extrasystoles were occasionally induced in the course of fasting. However, these findings clearly disappeared after the completion of the fasting. These transient abnormalities of ECG were observed in 9 patients $(3 \%)$ of all the total cases.

$X$-ray findings of upper GI. Only one case of conversion hysteria showed the formation of gastric ulcer after fasting. This finding indicated that the direct effect of fasting could be stressful enough to induce such a lesion.

Liver function. Checks of liver function were carefully made during and after the fasting therapy in fear of liver dysfunction due to fasting. As shown in Fig. 


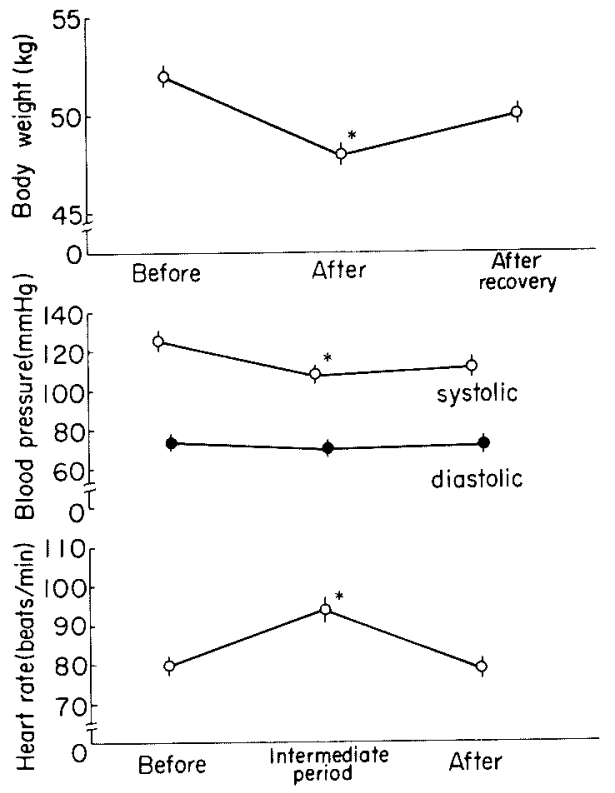

Fig. 1. Body weight, blood pressure and heart rate during fasting therapy. Each value is given as mean \pm s.E. $(n=50) p<0.001$.
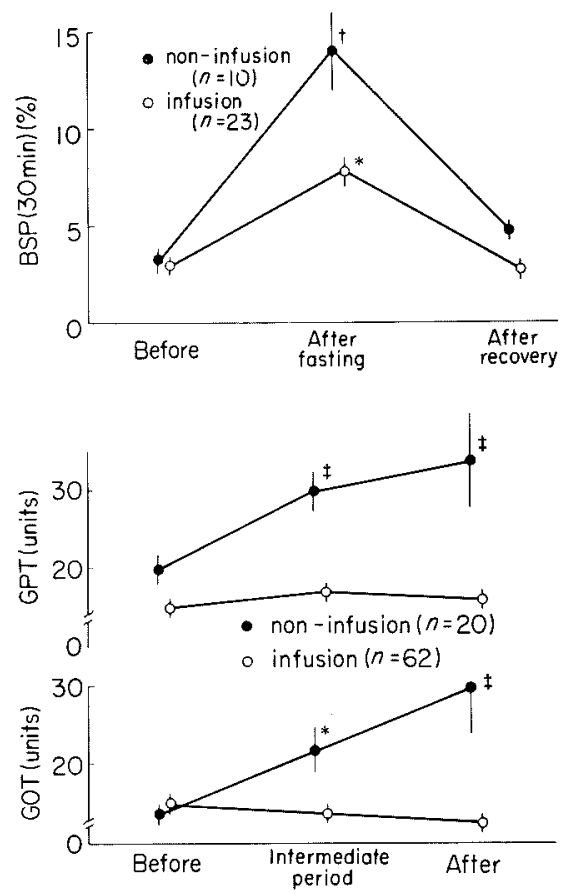

Fig. 2. Changes in liver functions during fasting therapy. Values are given as mean \pm S.E. $* p<0.02, \dagger p<0.01, \ddagger p<0.001$. 
2 , the results of liver function test were markedly different between a group without fluid supplement and another one with infusion; abnormally high BSP retention reached $14.0 \pm 6.7 \%$ at $30 \mathrm{~min}$ during fasting in the former group, while it was $7.9 \pm 3.4 \%$ in the latter group; both sGOT and sGPT showed a tendency to rise up in the group without fluid supplement. Even in the convalescence, such a rise was occasionally seen instead of a decrease. In contrast, scarcely any change was noted in the group with infusion. This is the very reason for the need of some amounts of fluid supplement containing carbohydrate during fasting.

Serum lipids, proteins and electrolytes. All the serum lipids examined such as total cholesterol, phospholipid and FFA rose significantly during fasting. They commonly showed a rise gradually from the middle of the fasting period and continued throughout fasting. The most conspicuous change was observed in serum FFA which rose up to about 5 times the prefasting level. As shown in Fig. 3 , most of them, except triglyceride, returned to their previous levels after the complete return to a normal diet.

Serum total protein fell slightly in the course of fasting and the level was almost unchanged till the end of fasting. This tendency was nearly the same as observed in serum electrolytes.

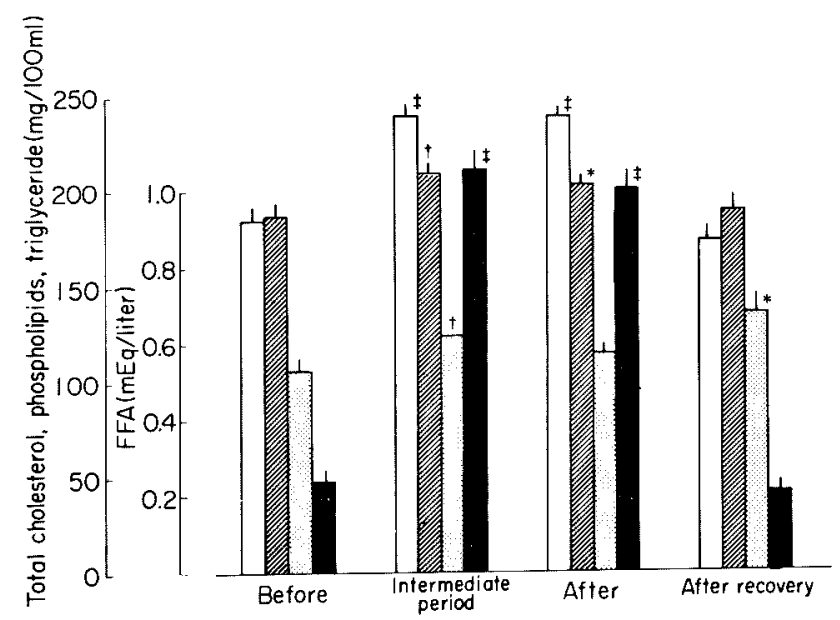

Fig. 3. Serum lipids levels during fasting therapy (mean \pm S.E., $n=37$ ) $\square$ total cholesterol; 圈 phospholipid, $\square$ triglyceride; free fatty acid.

${ }^{*} p<0.02, \dagger p<0.01, \ddagger p<0.001$. Vertical bar on each column indicates s.e.

Blood sugar, urinary ketone bodies, and catecholamines. As the fasting therapy advanced, a significant drop in blood sugar levels was generally noted. The lowest point $(63 \pm 11.3 \mathrm{mg} / 100 \mathrm{ml})$ was seen on the half way of the fasting therapy, from which the blood sugar values tended to rise on the 10th day, returning to the 
original levels after resuming meals. Throughout the period of fasting, however, no hypoglycemic sign was observed, whereas the urinary CA levels tended to rise since the earlier half up to the middle of the fasting process. This can be interpreted as an adaptability of living body for the hypoglycemia in order to maintain blood sugar homeostasis. As was shown in Fig. 4, levels of urinary CA excretion were relatively low throughout the entire period of the fasting, probably due to the resting state of the patients in fasting.

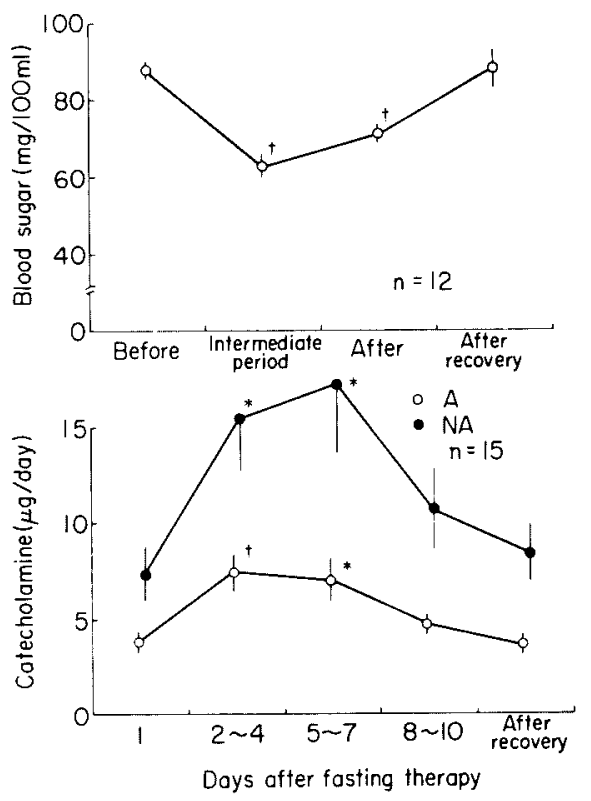

Fig. 4. Blood sugar levels and urinary catecholamine excretion during fasting therapy. A, adrenaline: NA, noradrenaline. mean \pm s.e. $* p<0.05, \dagger p<0.02$.

$E E G$. An examination for qualitative changes in EEG of 12 patients revealed no significant pathological finding except in a case of masked depression; she showed sporadic $14 \mathrm{c} / \mathrm{s}$ positive spikes mainly at the induction to occipital region on the 10th day of fasting. Generally, there was neither a burst of abnormal waves nor asymmetry in the wave, and the reactions to the photic stimulation and hyperventilation were found to be normal. Fig. 5 illustrates the effects of the therapy on the EEG, which was examined before and after the fasting therapy. This 18-year-old patient suffered from the hyperventilation syndrome. In the patient's regular waves on the initial day of the fasting therapy, an EEG frequency of $10 \mathrm{c} / \mathrm{s}$ was predominant, while on the 5th and 10th days, the frequency was lowered to $9 \mathrm{c} / \mathrm{s}$ and $8 \mathrm{c} / \mathrm{s}$, respectively; these slowed alpha-waves induced by fasting were irregular. The frequency peaked at $11 \mathrm{c} / \mathrm{s}$ with restored regularity of the waves after resuming meals. A similar tendency was observed in the wave amplitude of this case. Thus, the changes in the distribution of frequency of EEG 


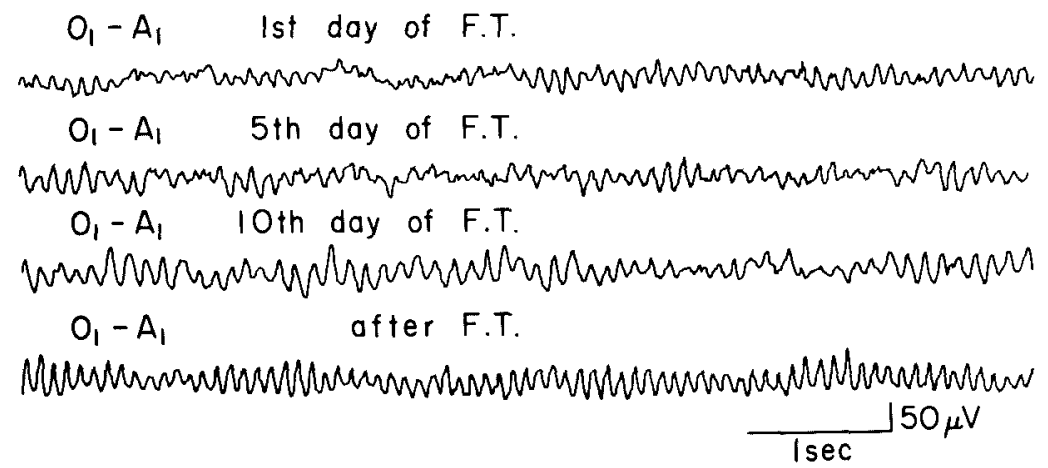

Fig. 5. EEG pattern during fasting therapy. T.U. (18-year-old), female, hyperventilation syndrome

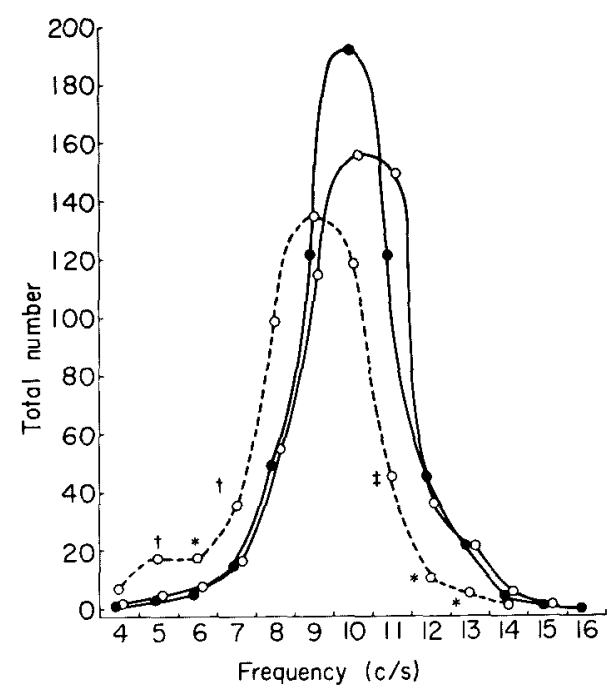

Fig. 6. Changes in frequency distribution of EEG waves during fasting therapy.

- lst day, 0 -.- 10 th day, $\bigcirc \circ$ after fasting therapy. ${ }^{*} p<0.05, \dagger p<0.02$, $\ddagger p<0.01, n=12$.

during fasting therapy were quantitatively examined. Fig. 6 shows the results of determination of total EEG frequencies in 12 patients on the first and the 10th days of fasting therapy and after resuming meals. As indicated by the closed circle, normal distribution with a peak at an EEG frequency of $10 \mathrm{c} / \mathrm{s}$ was observed initially, but slowed alpha-waves with an increase in the incidence of theta-waves following the fasting therapy could be seen, as shown by the open circle with broken line, which returned to a configuration strikingly similar to the original one when the fasting therapy was terminated. However, delicate changes were found, which were characterized by the slight increase in the $11 \mathrm{c} / \mathrm{s}$ alpha-waves as indicated by the open circle with a solid line. In order to examine a possible 
mechanism of these slowing of alpha-waves, a comparison was made between the first and the 10th days of the fasting therapy in terms of the drop in the blood sugar level and the decrease in the total number of $10 \mathrm{c} / \mathrm{s}$ alpha-waves. A significant positive correlation could be proven with a correlation coefficient of 0.74 , as shown in Fig. 7. This finding suggests that EEG wave patterns might be slowed down by low blood sugar levels induced by the fasting therapy.

Growth hormone. The serum GH was used as an index of possible neuroendocrinological changes during the fasting therapy. Fig. 8 illustrates the results of observations on the reaction of $\mathrm{GH}$ when the provocative tests were done

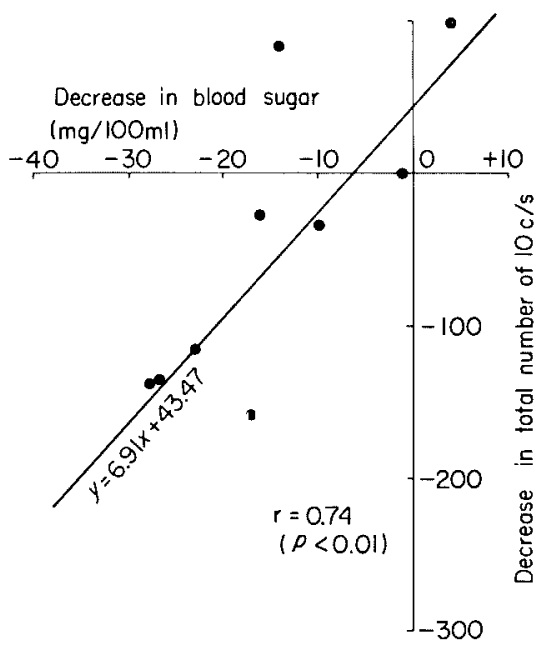

Fig. 7. Correlation between decrements of total number of $10 \mathrm{c} / \mathrm{s}$ waves and of blood sugar levels. $(n=12)$

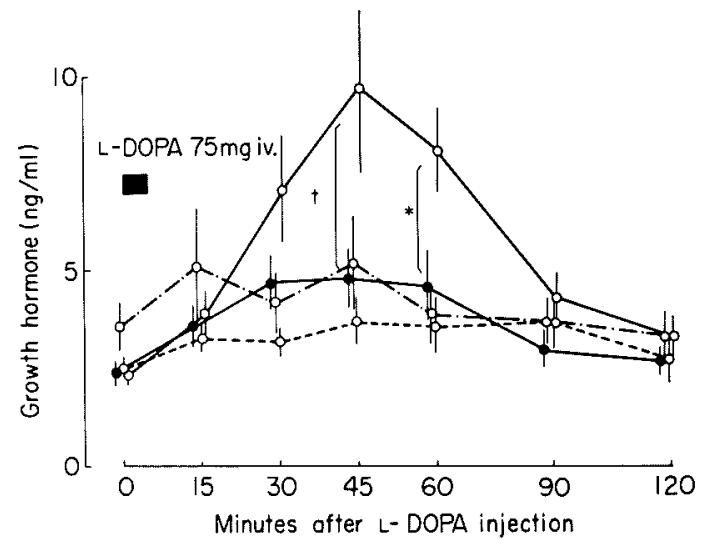

Fig. 8. L-DOPA test during fasting therapy.

- list day, $0-\cdot-0$ 5th day, $0 \cdots 0$ 10th day, $0-0$ after fasting therapy.

$* p<0.05, \dagger p<0.02$. Each value is mean \pm s.E. $(n=12)$. 
by L-DOPA in 10 patients. The average initial level of serum GH was 2.4 $\mathrm{ng} / \mathrm{ml}$, which rose to $4.8 \mathrm{ng} / \mathrm{ml}$ at $45 \mathrm{~min}$ after L-DOPA loading on the first day of the fasting therapy, but no significant change was observed in the course of the fasting period, although a slight increase in the initial GH level was observed. On the 10th day of fasting, the lowest levels of GH were noted, possibly due to generalized decreased synthesis of the hormone after the prolonged fasting. In contrast, the peak of the hormone level, $9.7 \mathrm{ng} / \mathrm{ml}$, was seen after resuming meals, at which time it was found to be higher than pretreatment levels depsite of rather wide scattering.

\section{Discussion}

Our findings confirm that total fasting for a period of 10 days can be practised without unfavorable effects on health. However, fasting for longer periods may result in some unwanted side effects; for example, some patients experienced such complications as hairloss, edema, muscular weakness, and in one case, polyneuritis in a study reported by Rooth and Carström (1970). In their study, small amounts of skim milk or lean meat were given to other patients to reduce the endogenous protein breakdown, who subsequently, exhibited no such side effects. The procedure is advisable only for the purpose of reducing body weight. However, from the viewpoint of psychosomatic treatment, any caloric intake may be undesirable even if they are negligible in nutrition, because it seems especially important for the patients to tolerate the hunger sensation or appetite, and to overcome painfulness through fasting.

Needless to say, the significance of our study on various bodily functions during fasting consists in the prediction of possible danger during the fasting period. Slight changes in liver functions are therefore worthy of attention. Although no jaundice occurred, significant elevations of transaminases as well as BSP retentions were observed. However, the changes were mostly transient and returned gradually to the initial levels after resuming meals. When small amounts of pentose solution with amino acids were parenterally given, the changes in liver functions diminished considerably. It is well known that the catabolic process of fat and protein is enhanced during prolonged fasting, which contributes to maintaining the normal blood sugar levels. It seems, therefore, that elevation of transaminases will result from the process (gluconeogenesis) which converts amino acids to glucose. This shows an adaptation of the human body which may be relieved by using a fluid supplement. On the other hand, the direct influence of fasting on the liver cells is also suspected, where an increase in permeability of the cell membranes may occur. Recently, however, the experiment on rats investigated by Nitarai et al. (1975) showed no significant change in sGOT, sGPT, alkaline phosphatase and aspirin esterase activity during fasting, in spite of a marked decrease in liver wet weight. Thus, some signs of liver dysfunction during fasting may be somewhat different from those of liver diseases clinically seen. It is evident from our results that a more or less ketotic state occurs due to the production of keto acids 
in the liver and/or the kidney. According to the investigation of Owen et al. (1969), these keto acids become the source of energy, some of which are utilized in the brain, representing a unique adaptation of human metabolism. However, care must be taken for patients who suffer from diabetes, because of a decreased glucose tolerance and possible aggravation of ketosis (Mzagournis and Skillman 1970; Yamauchi et al. 1972).

Our results showed that fasting therapy has relatively broad area of clinical application to patients suffering from various diseases. Based on our total results, this therapy may be the first choice to the pathological state with unfavorably conditioned reaction among many psychosomatic patients, because it has shown remarkable clinical effects in treating habituated symptoms such as aphonia, various kinds of tics (coughing tic, automatized belching attack and hiccup, etc.). Most of these symptoms, which had been refractory of other treatments, were relieved promptly after fasting. Excellent effects can be exerted also in the case of patients suffering from irritable colon, including some polysurgery cases in which iatrogenic factors have been involved (Yamauchi et al. 1970). This therapy is also recommended to the patients with lumbago having the mechanism of avoidance conditioning. The results will be satisfactory for the cases of autonomic nerve dystonia due to anxiety neurosis or conversion hysteria in the same sense. In addition, fasting will have beneficial effects on changing the abnormal eating behaviors in diabetic patients (Yamauchi et al. 1971) and in cases of dysorexia nervosa. Moreover, fasting therapy provides an option to the use of antidepressants and their accompanying side effects for the non-specific treatment of depression. Clinical effects would be generally small in the cases of cenesthopathy and of strongly distorted personality. Excellent results cannot be expected from poorly motivated patients. It is quite contraindicated to the older patients who have severe somatic disorders on the brain, heart and kidney, etc. (Suzuki et al. 1972). Although the fasting therapy is of wide application, it is not a panacea; it is important to determine the clinical indication of fasting therapy.

Another important problem with respect to fasting therapy is what is the nature of underlying mechanism of this therapy. The authors think the fasting therapy may be a kind of shock therapy, because it brings about a gradual development of hypoglycemia as well as many other physiochemical changes in the internal environments of human body. Some evidence supporting this veiw was obtained by us; i.e. significant drop of blood sugar, increase in heart rate, and increase in urinary CA levels were commonly observed. Presumably these changes in the internal environments might induce a stress-like condition in human body, to which, in turn, the body reacts physiologically. Thus, the ischemic changes (Kjellberg and Reizenstein 1970) or arrhythmias transiently recognized during fasting may be in part due to the stress-like condition. A patient, who developed a stomach ulcer after fasting, gives further evidence for such a claim. Consequently, the fasting therapy may be regarded as a kind of shock therapy, 
resulting in reorganizing the normal psychosomatic interaction. The theory may be further supported by delicate but significant changes in the EEG and GH during fasting.

The significantly slowed waves in EEG during fasting may suggest that the transient hypofunction of the CNS is induced by fasting. Although a similar slowing of EEG wave patterns is known to occur in a state of acute hypoglycemia (Kiloh et al. 1972), the two phenomena might differ in some ways. They may have the common effect to alter the brain functions, but the more integrated and broader potency seen in the prolonged fasting will lead the patients to a favorable state. It seems plausible that more complex changes in the autonomic nerves and the endocrinological centers can occur during the prolonged fasting. These specific actions of the fasting therapy may play an important role in extinction of abnormally formed conditionings in the brain. In connection with this, Adachi's experiments (1965) on EEG in rabbits will give valuable insights; he observed an inhibitory effect brought about by fasting on the hippocampus and hypothalamus without changes in the cortex and reticular formation. In addition, Kushima et al. (1963) reported an electron-microscopic examination of the hippocampal nerve cells of fasted rats; they clearly indicated the effects of fasting upon the cells, where such changes as the decrease in fine granules in the cytoplasm, undersized and pycnotized mitochondria, shortened endoplasmic reticulum, etc. were observed during fasting, but the morphological findings returned to the normal after breaking the fast. Summarizing these results, they attributed the altered structures and functions in the brain cells to the improvement of psychosomatic disorders in humans. These findings are very similar to our results related to the changes in serum GH response to L-DOPA loading during and after fasting; our investigation showed that the GH levels were generally low during fasting, but the grade of the reaction clearly rose after resuming meals. This finding is of great interest, particularly in light of the tendency of alpha-waves to peak at $11 \mathrm{c} / \mathrm{s}$ after resuming meals. The reason for the generalized hyporesponse of GH during fasting is not yet well understood, but it may be due to the gradual decrease in the CA pool in the CNS, whereas, not only the increase in CA synthesis in the CNS but also some changes in the sensitivity of $\mathrm{GH}$ releasing factor would mediate the hyperresponse of $\mathrm{GH}$ after resuming diet. However, there may arise some additional questions whether or not these GH responses are derived merely from L-DOPA itself. It is certain that some cases examined showed nausea-vomiting directly after the injection of L-DOPA, but they did not necessarily indicate the high levels of GH. In addition, the placebo study done by Singh et al. (1973) indicated that the observed rise in $\mathrm{GH}$ is not due to venipuncture nor to the nonspecific stress in the observed period. Thus, it appears that the neurocerebral functions are once inhibited by fasting, but are reactivated by the resumption of meals. This may suggest that a "new intracerebral equilibrium" has been established after the fasting therapy; it is especially noteworthy that a controlling of cortico-limbic and cortico-thalamo-hypothalamic systems can reorganize the 
disproportionation of neurocerebral functions and provide the patient with selfactualizing potentiality. Moreover, the prolonged fasting can give the patient a suitable place to deepen his insight into one's own disease. Even the changes in personality would be possible, if the therapists utilize the anaclitic situation under the fasting and apply some other methods as introspection or autogenic training.

On the other hand, the fasting condition may serve as either a type of aversive stimuli or punishment, if viewed from behavior therapy, and contribute to the destruction of old conditionings, because the fasting includes such instinctsuppressive procedures as deprivation of diet, freedom, and belongings from the patient. As was mentioned before, our experience showed that some cases with conversion reaction habituated with conditioing mechanism were promptly recovered by fasting therapy. In this respect, Murai and Sato (1965) demonstrated the effects of starvation on both extinction and acquisition of avoidance response in rats. They suggested the mechanism of the fasting therapy due to the observed facts of significant extinction of avoidance response facilitated by the fasting. Further experimental study indicated that the effects of starvation had lasted longer, even after a month of starvation (Sato and Murai 1966). These findings are in agreement with our findings on human subjects suggesting counter-conditioning in fasting. The salf-corfidence and pleasure of patients, when they overcome the agonizing limit-conditions can promote the relief of their symptoms owing to the biofeedback principle. In the future, it is hoped that more experiments, from the neurochemical as well as neuroendocrinological viewpoints, will be undertaken to determine the levels of monoamines and other hormones in the brain.

\section{Acknowledgment}

We wish to thank Dr. A. Ohnuma, Department of Pediatrics of Nagamachi Branch Hospital, and Dr. S. Ichijo, Neuropsychiatric Division of Sendai Tetsudo Hospital, for their valuable advice and instructions on EEG analyses. The growth hormone estimations were performed by Tokyo Special Reference Laboratory. This paper was presented in part at the 2nd and 3rd International Congress of Psychosomatic Medicine.

\section{References}

1) Adachi, K. (1965) The effective mechanism of hunger therapy and psychotropic drugs on psychosomatic disease in the electroencephalogram. J. Jap. Psychosom. Soc. (Jap.), 5, 219-226.

2) Bloom, W.L. (1959) Fasting as an introduction to the treatment of obesity. Metabolism, 8, 214-220.

3) Cahill, G.F. Jr., (1970) Starvation in man. New Engl. J. Med., 282, 668-675.

4) Cavagnini, F., Peracchi, M., Scotti, G., Raggi, U., Pontiroli, A.E. \& Bena, R. (1972) Effect of L-DOPA administration on growth hormone secretion in normal subjects and parkinsonian patients. J. Endocr., 54, 425-433.

5) Drenick, E.J., Swendseid, M.E., Blahd, W.H. \& Tuttle, S.G. (1964) Prolonged starvation as treatment for severe obesity. J. Amer. med. Ass., 187, 100-105.

6) Duncan, G.G., Jenson, W.K., Christofori, F.C. \& Schless, G.L. (1963) Intermittent fasts in the correction and control of intractable obesity. Amer. J. med. Sci., 245, 515-520. 
7) Hasegawa, N. (1961) Study of hunger therapy on psychosomatic disease. Sanfujinka no Zissai (Jap.), 10, 995-1008.

8) Kiloh, L.G., McComas, A.J. \& Osselton, J.W. (1972) Clinical Electroencephalography. 3rd ed. London Butterworths, p. 160.

9) Kjellberg, J. \& Reizenstein, P. (1970) Effect of starvation on body composition in obesity. Acta med. scand., 188, 177-178.

10) Kushima, K., Kamio, K., Hasegawa, N., Okazaki, T., Yamazaki, K. \& Takahashi, $\mathrm{K}$, (1959) Changes in fractions of urinary estrogen and 17-KS during fasting therapy on patients with psychogenic diseases. Clin. Endocr. (Jap.), 7, 703-707.

11) Kushima, K., Kamio, K. \& Hasegawa, N. (1961) Psychosomatic diseases in woman. Tohoku J. exp. Med., 74, 130-136.

12) Kushima, K., Kamio, N., Watanabe, T. \& Nakayama, C. (1963) Electron-microscopic studies on the nerve cells in hippocampus II. Changes during fasting. Brain and Nerve (Jap.), 15, 759-762.

13) Murai, N. \& Sato, T. (1965) The experimental study of the hunger therapy 1. Effect of starvation upon extinction and acquisition of avodiance response. Tohoku Psychol. Folia, 24, 38-45.

14) Mzagournis, M. \& Skillman, G. (1970) Glucose intolerance mechanism after starvation. Metabolism, 19, 170-178.

15) Nitarai, Y., Mitsui, S. \& Obara, K. (1975) Effects of starvation on pituitaryadrenal axis and hepatic function. J. Jap. Psychosom. Soc. (Jap.), 15, 280.

16) Ogawa, M. (1958) Significance of fasting therapy on psychosomatic diseases and endocrinological changes during fasting. Sanfuzinka no Sekai (Jap.), 10, 271-281.

17) Owen, O.E., Felig, P., Morgan, A.P., Wahren, J. \& Cahill, G.F., Jr. (1969) Liver and kidney metabolism during prolonged fasting. J. clin. Invest., 48, 574-583.

18) Rooth, G. \& Carström, S. (1970) Therapeutic fasting. Acta med. scand., 187, 455463.

19) Sato, T. \& Murai, N. (1966) The experimental study of the hunger therapy 3 . Effect of starvation upon extinction of conditioned emotional response. Tohoku Psychol. Folia, 25, 52-58.

20) Sato, T. (1971) Fluroimetric method for the determination of urinary catecholamines. Jap. J. clin. Path. (Jap.), 19, 333-336.

21) Singh, P., McDevitt, D.G., Mackay, J. \& Hadden, D.R. (1973) Effects of L-DOPA and chlorpromazine on human growth hormone and TSH secretion in normal subjects and acromegalics. Hormone Res., 4, 293-301.

22) Suzuki, J., Yamauchi, Y., Horikawa, M., Tamabuchi, Y. \& Makabe, M. (1972) Method and effect of fasting therapy. J. Jap. Psychosom. Soc. (Jap.), 12, 290-295.

23) Takihara, H. (1930) Study on Starvation (Jap.), Iwanami shoten, Tokyo. pp. $173-387$.

24) Thomson, T.J., Runcie, J. \& Miller, V. (1966) Treatment of obesity by total fasting for up to 249 days. Lancet, 2, 992-996.

25) Yamauchi, H. (1959) Study on serum serotonin in gynecological diseases. Clin. Endocr. (Jap.), 7, 714-719.

26) Yamauchi, Y., Suzuki, J. \& Horikawa, M. (1970) Three polysurgery cases and the clinical effects of hunger therapy. J. Jap. Psychosom. Soc. (Jap.), 10, 175-187.

27) Yamauchi, Y., Suzuki, J., Tamabuchi, Y. \& Horikawa, M. (1971) Psychosomatic aspects of diabetes mellitus. J. Jap. Psychosom. Soc., (Jap.), 11, 306-316.

28) Yamauchi, Y., Suzuki, J., Tamabuchi, Y., Hasegawa, N., Horikawa, M. (1972) Significance of fasting therapy on psychosomatic patients with diabetes. J. Jap. Soc. Intern. Med. (Jap.), 61, 832-833.

29) Yoshinaga, K. (1960) The estimation of catecholamines in urine for clinical use. Nittshinigaku (Jap.), 47, 775-783.

30) Yoshimoto, I. (1965) 40 years-study on Introspection (Jap.), Syunjusha, Tokyo. 